\title{
Comprehensive Management of Congenital Cataract in Dizygotic Twin Babies
}

\author{
Authors: \\ Windya Tri Hapsari*涫 \\ Indri Wahyuni ${ }^{\circ}$ \\ Rozalina Loebis
}

Affiliations:

Department of Ophthalmology, Faculty of Medicine, Universitas Airlangga-RSUDDr. Soetomo Surabaya, Indonesia.

Corresponding author: Windya Tri Hapsari windya.tri.hapsari-2019@ fk.unair.ac.id

\section{Dates:}

Received: 20 July 2021

Revised: 04 October 2021

Accepted: 05 October 2021

Published: 04 November 2021

DOI:

https://doi.org/10.20473/

vsehj.v1i1.2021.17-21

\section{Copyright:}

(C) 2021 Author(s). Open access under Creative Commons Attribution-Share Alike $4.0 \quad$ International Licence (CC-BY-SA).

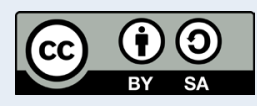

\begin{abstract}
Introduction: One of the leading causes of infant blindness is cataract. The prevalence of congenital cataract has been estimated in the range between 1 and 15 per 10.000 children globally. Congenital cataract in twin babies is a rare case. This case will report the outcome of comprehensive management of congenital cataract in dizygotic twin babies. Case presentation: Eight months old dizygotic twin babies came to our clinic with the primary complaint of whitish appearance in the pupil of both eyes since birth. They were born aterm with cesarean delivery. The birth weight was $2.700 \mathrm{~g}$ and $3.100 \mathrm{~g}$ respectively. They were the fourth and fifth children in the family. There was no family history of congenital cataract. The visual acuity of both eyes was positive response to light stimuli. The cataract was bilateral and dense on both babies. Fundus reflex and TORCH examinations were negative. Ultrasonography (USG) of the retina was normal on both babies. Conclusions: Isolated congenital cataract in twin babies is an unusual condition that should be treated comprehensively. Congenital cataract may cause deprivation amblyopia, refractive amblyopia, and permanent vision impairment. Early diagnosis and quick treatment, such as surgical timing and visual rehabilitation, are critical to perform successful management. Comprehensive care is required to monitor the visual result of cataract surgery.

Keywords: congenital cataract; dizygotic twin babies; visual rehabilitation
\end{abstract}

\section{Introduction}

Pediatric cataract is one of the leading causes of avoidable childhood blindness, affecting over 200.000 children globally. Congenital cataract has been found to affect 1 to 15 per 10.000 children globally, with an estimated frequency of three to six per 10.000 live births. ${ }^{[1]}$ The incidence in the United States is 2.0 per 10.000 births. ${ }^{[1]}$ In China, congenital cataract cases are about 5.0 per 10.000 births, and $22-30 \%$ of childhood blindness is attributed to congenital cataract in the absence of appropriate treatment. Delayed presentation to hospital and late surgical treatment being the significant causes of blindness and visual impairment. ${ }^{[2]}$ Congenital cataract etiology was hereditary origin, particular metabolic diseases, accompanying ocular abnormalities or systemic findings. Optical correction for congenital cataracts with aphakic glasses, contact lenses, or Intraocular Lenses (IOL) implantation was strongly suggested. ${ }^{[1],[2],[3]}$

Isolated congenitalcataractindizygotictwininfantsisunusualtofind.Theclinical condition, initial therapy, and follow-up visual rehabilitation management are all covered in this case report. This condition necessitates comprehensive therapy. Early identification and quick treatment result in a favorable visual outcome.

\section{Case presentation}

Eight-month-old dizygotic twin baby boys came to our clinic with complaints of whitish appearance in the pupil of both eyes since birth. There was also nystagmus on both babies since they were three months old. These babies were born at term with section cesarean delivery. The birth weight was $2.700 \mathrm{~g}$ and 
$3.100 \mathrm{~g}$, and they were the fourth and fifth children in the family. There was no history of congenital cataract in the family. The visual acuity of the right and left eye was fix and follow with light stimuli. Anterior segment examination reveals a dense bilateral cataract on both babies (Figure 1). Fundus reflex and posterior segment of the eyes were unable to evaluate. TORCH serology examination was negative, and Ultrasonography (USG) of both eyes was normal on both babies (Figure 2).

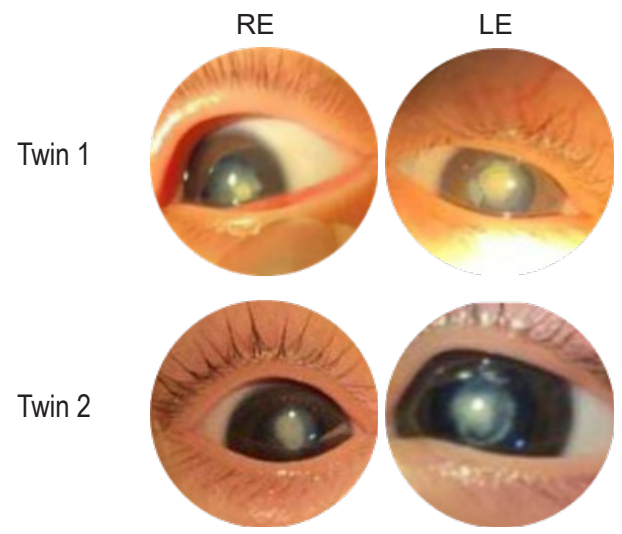

Figure 1. Twin anterior segment; the cataract was bilaterally and dense on both eyes.

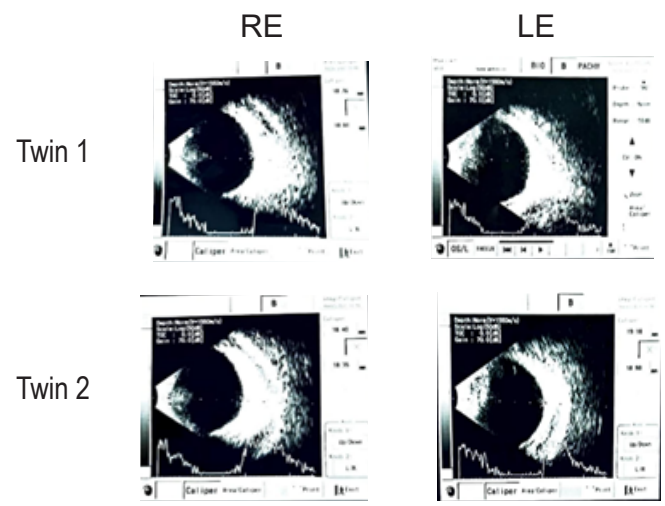

Figure 2. Twin USG for both eyes were normal.

Cataract surgeries on both babies were performed with the technique of lensectomy without IOL implantation. Examination under anesthesia was done after the first cataract surgery of these babies. Right eye (RE) horizontal and vertical corneal diameters of the first twin were $11.5 \mathrm{~mm}$ and left eye (LE) horizontal and vertical corneal diameters were $11 \mathrm{~mm}$. Furthermore, the cornea of both twin babies was normal. Intra Ocular Pressure (IOP) of the first twin RE and LE was $14.6 \mathrm{mmHg}$. In the second twin $\mathrm{RE}$ intra ocular pressure was $12.2 \mathrm{mmHg}$ and LE intra ocular pressure was $17.3 \mathrm{mmHg}$. Keratometry result of the first twin RE was K1 7.99/K2 7.50 and LE was K1 8.10/ K2 7.58. In the second twin RE keratometry result was K1 6.92/K2 6.41 and LE keratometry result was K1 7.58/K2 7.12. Biometry results of both eyewas shown in Table 1. After first surgery, both left eyes were aphakia (Figure
3). Streak retinoscopy result of the first twin LE was +16.00 D and the second twin LE was +17.00 D. Anterior segment examination of both LE babies revealed fibrotic posterior capsule in central and posterior polaris cataract. Posterior segment examination of both LE babies was normal.

After second surgery both RE babies became aphakia (Figure 4), these twin babies' aphakic glasses' visual acuity were $6 / 36$ and $6 / 48$. This result was within normal limit for their age. Visual rehabilitation started with quick post-surgical medications. These twin babies were treated with tobramycin antibiotic and dexamethasone steroid eyedrop every four hours for the right eye dan every six hours for the left eye, anticholinergic homatropine $2 \%$ eyedrop two drops every eight hours for both eyes. Oral medication of dexamethasone $0.5 \mathrm{mg}$ tablet was given every eight hours orally. Paracetamol syrup 5 $\mathrm{ml}$ was given every eight hours orally. Optical visual rehabilitation for these babies was aphakic glasses with the prescription for the first twin was $+16.00 \mathrm{D}$ and the second twin was $+17.00 \mathrm{D}$. Since these babies were only eight months old, implantation of intraocular lens was postponed until they become two years old to minimize complications and optimize the result of visual outcome.

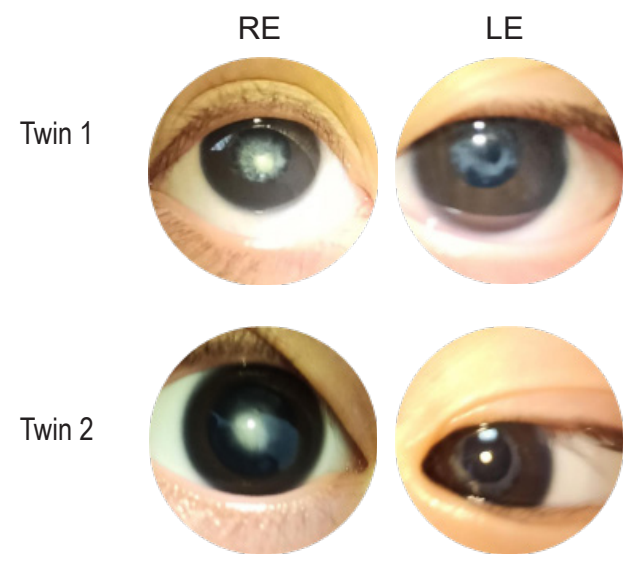

Figure 3. The twin anterior segment in the first operation with right eyes were opaque lens, and left eyes were aphakia.

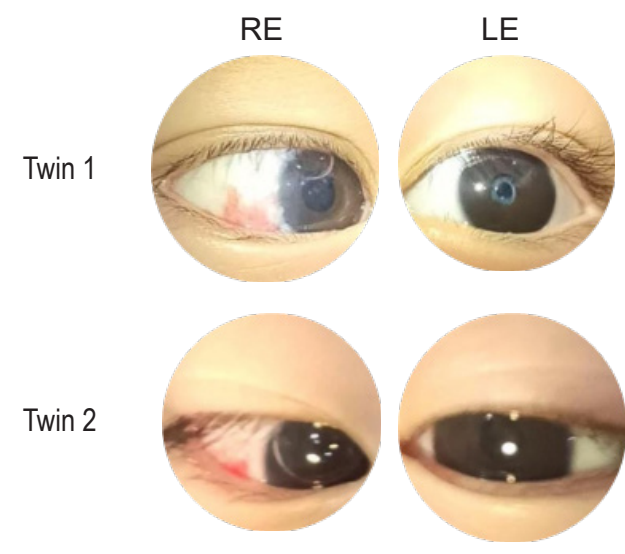

Figure 4. The twin anterior segment in the second operation with right and left eyes was aphakia. 
Table 1. The biometry of the twin.

\begin{tabular}{|c|c|c|c|c|c|c|c|c|c|c|c|}
\hline \multicolumn{6}{|c|}{ Twin 1} & \multicolumn{6}{|c|}{ Twin 2} \\
\hline \multicolumn{2}{|c|}{$\mathrm{RE}$} & & \multicolumn{3}{|c|}{ LE } & \multicolumn{2}{|r|}{$\mathrm{RE}$} & & \multicolumn{3}{|c|}{ LE } \\
\hline \multicolumn{2}{|c|}{$\mathrm{K} 1: 7.99 \mathrm{~mm}$} & & \multicolumn{3}{|c|}{$\mathrm{K} 1: 8.10 \mathrm{~mm}$} & \multicolumn{2}{|c|}{$\mathrm{K} 1: 6.92 \mathrm{~mm}$} & & \multicolumn{3}{|c|}{$\mathrm{K} 1: 7.58 \mathrm{~mm}$} \\
\hline \multicolumn{2}{|c|}{$\mathrm{K} 2: 7.50 \mathrm{~mm}$} & & \multicolumn{3}{|c|}{$\mathrm{K} 2: 7.58 \mathrm{~mm}$} & \multicolumn{2}{|c|}{$\mathrm{K} 2: 6.41 \mathrm{~mm}$} & & \multicolumn{3}{|c|}{$\mathrm{K} 2: 7.12 \mathrm{~mm}$} \\
\hline \multicolumn{2}{|c|}{ AXL: $20.73 \mathrm{~mm}$} & & \multicolumn{3}{|c|}{ AXL: $20.74 \mathrm{~mm}$} & \multicolumn{2}{|c|}{ AXL: $20.89 \mathrm{~mm}$} & & \multicolumn{3}{|c|}{ AXL: $21.56 \mathrm{~mm}$} \\
\hline \multicolumn{2}{|c|}{ ACD: $2.61 \mathrm{~mm}$} & & \multicolumn{3}{|c|}{ ACD: 3.73 mm } & \multicolumn{2}{|c|}{ ACD: $3.31 \mathrm{~mm}$} & & \multicolumn{3}{|c|}{ ACD: 3.22 mm } \\
\hline \multicolumn{2}{|c|}{ LT: 1.59 mm } & & \multicolumn{3}{|c|}{ LT: $2.31 \mathrm{~mm}$} & \multicolumn{3}{|c|}{ LT: 1.91 mm } & \multicolumn{3}{|c|}{ LT: 2.01 mm } \\
\hline \multirow[t]{3}{*}{$\mathrm{SRK} / \mathrm{T}$} & 28.50 & 0.48 & SRK/T & 29.00 & 0.45 & $\mathrm{SRK} / \mathrm{T}$ & 21.50 & 0.17 & SRK/T & 23.50 & 0.28 \\
\hline & 29.00 & 0.10 & & 29.50 & 0.07 & & 22.00 & -0.16 & & 24.00 & -0.08 \\
\hline & 29.50 & -0.28 & & 30.00 & -0.31 & & 22.50 & -0.48 & & 24.50 & -0.44 \\
\hline \multirow[t]{3}{*}{ Hoffer Q } & 29.50 & 0.55 & Hoffer Q & 30.50 & 0.23 & Hoffer Q & 20.50 & 0.40 & Hoffer Q & 23.50 & 0.41 \\
\hline & 30.00 & 0.18 & & 31.00 & -0.15 & & 21.00 & 0.07 & & 24.00 & 0.05 \\
\hline & 30.50 & -0.20 & & 31.50 & -0.53 & & 21.50 & -0.27 & & 24.50 & -0.30 \\
\hline \multirow[t]{3}{*}{ Holladay } & 29.50 & 0.22 & Holladay & 30.00 & 0.25 & Holladay & 20.00 & 0.32 & Holladay & 23.50 & 0.35 \\
\hline & 30.00 & -0.15 & & 30.50 & -0.13 & & 20.50 & -0.02 & & 24.00 & 0.00 \\
\hline & 30.50 & -0.53 & & 31.00 & -0.51 & & 21.00 & -0.36 & & 24.50 & -0.36 \\
\hline \multirow[t]{3}{*}{ Haigis } & 31.50 & 0.34 & Haigis & 33.50 & 0.43 & Haigis & 21.50 & 0.48 & Haigis & 25.00 & 0.48 \\
\hline & 32.00 & -0.02 & & 34.00 & 0.07 & & 22.00 & 0.14 & & 25.50 & 0.13 \\
\hline & 32.50 & -0.39 & & 34.50 & -0.29 & & 22.50 & -0.20 & & 26.00 & -0.22 \\
\hline
\end{tabular}

\section{Discussion and conclusions}

Congenital or infantile cataracts are those that appear at birth or develop within the first year of life. Congenital cataract in twin infants is a rare disease that should be investigated. About one-third of congenital cataract cases are inherited without a systemic problem-congenital hereditary cataracts in a dizygotic twin pregnancy with body weight and affected status differences between two fetuses. One of the leading causes of avoidable juvenile blindness is a pediatric cataract. Congenital cataracts are categorized according to their appearance, assumed or established genetic origin, particular metabolic abnormalities, or accompanying ocular defects or systemic symptoms. Some known causes of congenital and infantile cataracts include rubella, cytomegalovirus, varicella-herpes zoster, herpes simplex, toxoplasmosis, syphilis, Epstein-Barr virus, measles, poliomyelitis, intrauterine drug exposure, intrauterine ionizing radiation, a prenatal or perinatal metabolic disorder such as galactosemia. Hereditary with a systemic disease or multisystem dysmorphic syndrome, such as chromosomal trisomy 21, Turner's syndrome, trisomy 1315 , trisomy $16-18$, deletion chromosome 5 , or idiopathic (without a recognized cause). The twin chromosomal examinations were standard this case, and the TORCH examination results were negative on both babies. Furthermore, we can conclude that the cataract on these dizygotic twin babies was isolated. Parent education should be given to assure the success of the surgical results. Parents should be told that surgery is only the beginning and that long-term follow-up and compliance with all visual rehabilitation care, including the use of aphakic glasses and occlusion therapy with an eye patch, is required. Appropriate postoperative care is strongly advised, including a rapid optical correction in the form of aphakic glasses or contact lenses or IOL implantation at the appropriate age ( $>2$ years). The timing of surgery is critical for the prognosis of congenital cataracts. It has also been reported that the presence of other associated ocular abnormalities, such as nystagmus, strabismus, Persistent Hyperplastic Primary Vitreous (PHPV), Congenital Persistent Pupillary Membrane (CPPM), small eyes, and minor corneal disorders, has a significant effect on prognosis. Amblyopia therapy and frequent follow-up exams should be initiated as soon as feasible to ensure a favorable visual result after surgery. ${ }^{[1],[2],[3],[4],[5],[6],[7],[8]}$

We chose a lensectomy technique without intraocular lens implantation for these dizygotic twin babies because the baby's eyes have short axial lengths, shallow anterior chambers, corneal curvature changes, and corneal thickness changes in children under two years old, which leads to higher prediction errors. Prediction errors have been attributed to a variety of factors, including inaccuracies in axial length measurements in the supine position, biometry technique, keratometry values, the phenomenon of pseudo accommodation, variability in IOL position due to capsule fibrosis, and increased corneoscleral elasticity in children. ${ }^{[3]}$ 


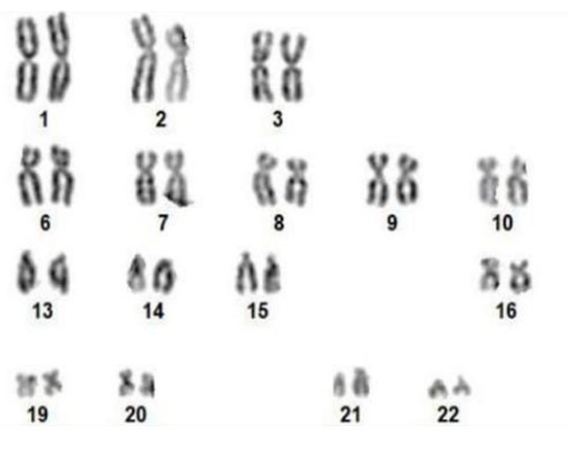

Twin 1
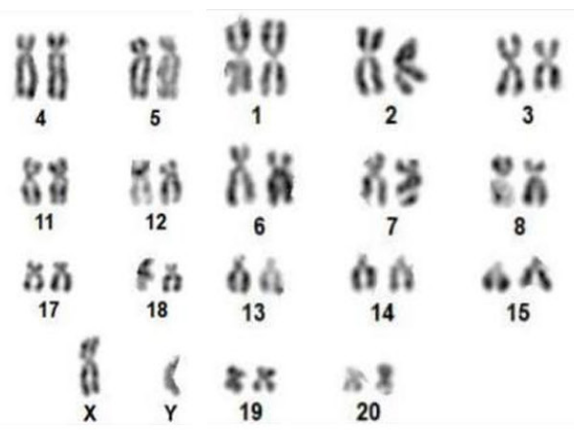

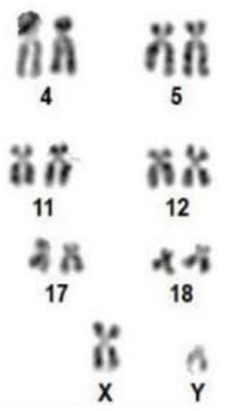

Twin 2

Figure 5. Twin chromosomal examination was normal; The first twin there are 46-XY, in the 20 cells examined; and the second twin there are 46-XY, in the 15 cells examined.

Visually significant unilateral cataracts should be extracted at six weeks of age to improve visual outcomes, and bilateral cataracts should be extracted between six and eight weeks of age, with 1-2 weeks interval between lensectomy for each eye. These dizygotic twin infants will be implanted with IOLs once they reach the age of two. The rationale for this is sufficient capsular support after two years of age and a lower chance of prediction mistakes. Cataract surgery in children has been quickly developing because of better surgical methods, equipment, and novel IOL designs. Due to average ocular growth, the limited prediction of lens calculators for shorter eyes, and the difficulty of achieving IOL parameters in conscious children, IOL selection in youngsters is problematic. Current lens formulae need axial length and corneal curvature $(\mathrm{K})$ data to estimate the final refractive result. Patients who are young and recalcitrant and require anesthesia for biometric assessment are more likely to have higher biometric inaccuracy due to a lack of fixation. It has been observed that in eyes with a shorter axial length and a steeper cornea, the Hoffer- $Q$ formula is more sensitive to biometric errors than other theoretical formulae like SRK/T, Holladay I, and Haigis formula. However, SRK/II has an invariable sensitivity to the magnitude of the biometric mistake. Nihalani and Van der Veen reported that Hoffer-Q $\mathrm{Q}$ is the most predictive formula among patients who underwent IOL implantation for pediatric cataracts before 18 years. However, Lee et al. study ${ }^{[4]}$ reported that SRK/II was the most predictive formula across all age subgroups, and Hoffer- $Q$ was the least predictive for patients who received IOL implantation surgery during their first decade. The Lee et al. research ${ }^{[4]}$ found a substantial connection between age at IOL implantation and prediction error derived by the SRK/II, SRK/T, and Hoffer-Q formulas.

After ten years, all three formulas had the same level of predictability; however, of the three formulas tested, the SRK/ II formula consistently delivers the best meaningful prediction in individuals with congenital cataracts before ten. Most pediatric surgeons place hydrophobic one-piece acrylic lenses in the capsular bag or threepiece lenses in the ciliary sulcus. Although multifocal and accommodating lenses can give good distant and close vision, myopia in newborns inhibits the refractive precision required for proper IOL selection. Most ophthalmologists now utilize IOLs to treat surgical aphakia after the age of two. A frequent consequence of juvenile cataract surgery is visual axis opacity. In order to decrease the high prevalence of visual axis opacity, lensectomy in children under the age of four usually comprises posterior capsulotomy with minimal anterior vitrectomy.

Using aphakic glasses based on the residual refractive error of these dizygotic twin infants results in an excellent visual outcome. These babies have visual acuity 4 CPCM $(57 \mathrm{~cm}) \sim 4 \mathrm{cpd} \sim 6 / 36-6 / 48$. Education to parents about the importance of wearing the aphakic glasses in all of the baby activity time is essential to improve the visual outcome and reduce the sensory deprivation amblyopia in these babies. The sensory nystagmus of these babies was reduced to normal after wearing the aphakic glasses. We plan to implant the IOLs when these babies become two years old to enhance satisfactory visual outcomes. In children with congenital cataracts, a primary IOL will provide satisfactory structural and functional outcomes. The timing of presentation and postoperative compliance to amblyopia therapy has an impact on the visual result. In addition to IOL implantation, it is critical to carefully monitor and correct the refractive error in the postoperative phase to avoid amblyopia. It is why we provide aphakic spectacles to these twin newborns for their optical visual rehabilitation. These dizygotic twin babies were also undergoing a chromosomal examination to detect morphology causes of hereditary congenital cataracts. Fortunately, their chromosomal examination is regular. Comprehensive management is needed for the case of these twin babies to follow up the visual outcome and visual rehabilitation after cataract surgery. Comprehensive management can provide a satisfactory

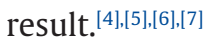


Isolated congenital cataract in dizygotic twin babies is a rare case. Congenital cataracts produce deprivation amblyopia, refractive amblyopia and lead to lifelong visual impairment. Awareness of the specific causes and visual condition of the twin babies must be diagnosed and treated earlier. Appropriate and comprehensive management will provide a satisfactory visual outcome. ${ }^{[8]}$

\section{References}

[1] American Academy of Ophthalmology. 2020-2021 BCSC (Basic and Clinical Science Course), Section 11: Lens and Cataract. San Fransisco: American Academy of Ophthalmology; 2020.

[2] Lin H, Yang Y, Chen J,ZhongX, LiuZ, LinZ, etal.Congenital cataract: prevalence and surgery age at Zhongshan Ophthalmic Center (ZOC). PLoS One 2014;9:e101781. https://doi.org/10.1371/journal.pone.0101781.

[3] Medsinge A, Nischal KK. Pediatric cataract: Challenges and future directions. Clin Ophthalmol 2015;9:7790. https://doi.org/10.2147/OPTH.S59009.

[4] Lee BJ, Lee S-M, Kim JH, Yu YS. Predictability of formulae for intraocular lens power calculation according to the age of implantation in paediatric cataract. Br J Ophthalmol 2019;103:106-111. https:// doi.org/10.1136/bjophthalmol-2017-311706.

[5] Yangzes S, Kaur S, Gupta PC, Sharma M, Jinagal J, Singh $\mathrm{J}$, et al. Intraocular lens implantation in children with unilateral congenital cataract in the first 4 years of life. Eur J Ophthalmol 2019;29:304-308. https://doi. org/10.1177/1120672118790193.

[6] Lim ME, Buckley EG, Prakalapakorn SG. Update on congenital cataract surgery management. Curr Opin Ophthalmol 2017;28:87-92. https://doi.org/10.1097/ ICU.0000000000000324.

[7] Rajavi Z, Sabbaghi H. Congenital Cataract Screening. J Ophthalmic Vis Res 2016;11:310-312. https://doi. org/10.4103/2008-322X.188389.

[8] Lee KA, Park M-H, Kim YJ, Chun SH. Isolated congenital hereditary cataract in a dizygotic twin: Prenatal ultrasonographic diagnosis. Twin Res Hum Genet Off J Int Soc Twin Stud 2013;16:994-997. https://doi.org/10.1017/thg.2013.50. 